\title{
Effect on Lanshi Tonic in Mice with Kidney-Yang Deficiency
}

\author{
Shuo Liu ${ }^{1}$, Si-yao $\mathrm{Ma}^{1}$, Wenyi Zhu ${ }^{2}$, Jia Liang ${ }^{3}$, Yu-jing Shi ${ }^{4}$, Hong Meng ${ }^{2 *}$ \\ ${ }^{1}$ Research and Development of Healthy Food, Infinitus (China) Co., Ltd., Guangzhou, China \\ ${ }^{2}$ Cosmetics Science and Technology, Beijing Technology and Business University, Beijing, China \\ ${ }^{3}$ Traditional Chinese Medicine, Graduate School of Chinese Academy of Traditional Chinese Medicine, Beijing, China \\ ${ }^{4}$ Institute of Chinese Materia Medica, China Academy of Chinese Medical Sciences, Beijing, China
}

*Corresponding author: Hong Meng, Cosmetics Science and Technology, Beijing Technology and Business University, No. 11/33, Fucheng Road, Haidian District, Beijing, China

Tel.: +861061374229

Email: menghong2000@163.com

Received May 09, 2020

Revised May 26, 2020

Accepted June 09, 2020

Published June 30, 2020

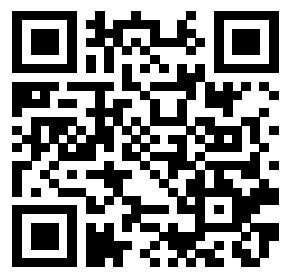

\begin{abstract}
Purpose: To study the regulatory effect of Lanshi tonic on kidney-yang deficiency model and its related mechanism. Methods: ICR mice were randomly divided into five groups according to body weight: normal group, model group, Lanshi tonic high, medium and low dose group. Mice with kidney-yang deficiency syndrome were induced by hydrocortisone sodium succinate by intraperitoneal injection, $50 \mathrm{mg} /$ $\mathrm{kg}$ for 7 days. At the same time, all groups were given corresponding medicine by oral administration for 10 days. The body mass, hair color and body shape of mice were observed and recorded. Spleen index, thymus index and adrenal index were calculated. Level of urine 17-OHCS was measured by ELISA. Level of serum Cortisol, ACTH, CAMP, cGMP and ratio of CAMP/CGMP were measured by ELISA. Results: Compared with the normal group, the above indexes of the model group mice were significantly lower ( $p<0.05, p<0.01$ ), except the serum cGMP was significantly higher $(p<0.01)$. Compared with the model group, Lanshi tonic at high and medium doses had no significant change in urine 17-OHCS content $(p>0.05)$, but had different improvement effects on the other indicators mentioned above $(p<0.05, p<0.01)$. Conclusion: Lanshi tonic can regulate the state of kidney-yang deficiency, which may be related to the regulation of adrenocortical secretion function and the imbalance of cyclic nucleotide levels.
\end{abstract}

Keywords: Lanshi tonic, Hydrocortisone sodium succinate, Kidney-yang deficiency, Adrenal cortex, Cyclic nucleotide

\section{Introduction}

肾阳虚是肾阳虚衰, 温煦失职, 气化失权所表现的一类虚 寒证候。当处于肾阳虚状态时, 全身机能降低, 代谢下降, 体 温降低, 免疫功能减退, 腰膝酸软, 易疲劳等 (Zhang et al., 2008; Xu et al., 2015)。现代医学研究表明, 肾阳虚证与神 经-内分泌-免疫系统的功能变化密切相关, 下丘脑-垂体-靶腺 轴 (肾上腺轴和性腺轴) 的功能障碍或低下是肾阳虚证的基础 (Shen, 1997)。

澜仕口服液由黄芪、杜仲、生地黄、巴戟天、枸杞子和黄精 组成, 具有增强免疫力、缓解体力疲劳的功效。本研究采用经 典的氢化可的松法制作肾阳虚小鼠模型 (Chen, 1993; Lin et al., 2013; Jia et al., 2016), 通过观察澜仕口服液对一般
体征状态、免疫器官指数、肾上腺皮质功能及环核苷酸水平的 影响, 探讨澜仕口服液对肾阳虚状态的调节作用。

\section{Methods}

\section{Instruments and materials}

1) Test instrument

全自动多功能酶标仪 (MULTISKAN MK3; 美国Thermo公司, 美 国); 电热恒温培养箱 (DH4000A; 天津泰斯特公司, 中国); 低温离心机 (5804R; 德国Eppendorf公司, 德国); MINI shaker (MH-1; Kylin-Be11 Lab Instruments QILINBEIER公司, 中国) 
2) Material

澜仕口服液 (批号18G04BAB01; 无限极 (中国) 有限公司, 中 国); 注射用氢化可的松琥珀酸钠（批号021703067, 天津生物 化学制药有限公司产品, 中国); Mouse Cortisol ELISA kit (CSB-E05113m; 武汉华美生物工程有限公司, 中国); Mouse ACTH ELISA kit (CSB-E06874m; 武汉华美生物工程有限公司, 中国); Mouse 17-0HCS ELISA kit (20181101; 南京建成生物工 程研究所, 中国); Mouse cAMP ELISA kit (20181024; 南京建 成生物工程研究所, 中国) ; Mouse cGMP ELISA kit（20181024, 南京建成生物工程研究所, 中国)。

\section{3) Animal}

ICR小鼠（SPF级）, 雌雄兼用, 体重为18-20 g, 购自北京华 阜康生物科技股份有限公司, 动物合格证号： 11401300086884 , 使用许可证号：SCXK（京）2015-0041。

\section{Experimental method}

\section{1) Animal grouping and treatment}

按体质量将小鼠随机分为5组: 正常组、模型组、澜仕口服 液高、中、低剂量组, 每组20只, 雌雄各半。除正常组外, 各 组小鼠给予氢化可的松琥珀酸钠 $50 \mathrm{mg} / \mathrm{kg}$ 腹腔注射, $0.1 \mathrm{~mL} / 10$ $\mathrm{g}$, 每天 1 次, 连续 7 天, 停止注射 3 天造成肾阳虚证候小鼠模 型。每日给予氢化可的松琥珀酸钠后间隔 2 小时给予相应药物灌 胃 $0.2 \mathrm{~mL} / 10 \mathrm{~g}$, 连续 10 天, 正常组和模型组在同等条件下给予 蒸馏水。本实验中使用小鼠等效剂量 $5.5 \mathrm{~mL} /(\mathrm{kg} \cdot \mathrm{d})$ 作为澜仕 口服液中剂量, 使用 2 倍和 $1 / 2$ 倍小鼠等效剂量分别作为澜仕口 服液高剂量和澜仕口服液低剂量。实验期间各组小鼠自由进食 进水。

2) Observation of general physical signs

每天观察大鼠饮食饮水情况、精神状态、毛色、体态, 末次 给药后称取体质量。

\section{3) Detection of 17-hydroxycorticosteroids of urine}

末次给药后收集 24 小时尿液, 离心取上清, $-20^{\circ} \mathrm{C}$ 保存待测, 采用 enzyme-linked immunosorbent assay (ELISA) 方法检测尿
17-羟皮质类固醇（17-hydroxycorticosteroid，17-0HCS）。

\section{4) Detection of ACTH/ Cortisol/ cAMP/ cGMP}

尿液收集结束后眼眭取血, 离心分离血清, $-20{ }^{\circ} \mathrm{C}$ 保存待测, 采用ELISA方法检测血清促肾上腺皮质激 素 (adrenocorticotropic hormone, ACTH) 、皮质醇 (cortisol) 、环磷酸腺苷 (cyclic adenosine monophosphate, cAMP) 、环磷酸鸟苷 (cyclic guanosine monophosphate, cGMP) 的表达。

5) Detection of organ index

解剖小鼠, 称脾重、胸腺重、肾上腺重, 计算脾指数、胸 腺指数、肾上腺指数。脏器指数及脏器指数抑制率计算公式如 下:

脾指数 $(\%)=[$ 脾湿重 $(\mathrm{g}) /$ 体重 $(\mathrm{g})] \times 100$

胸腺指数 $(\%)=[$ 胸腺湿重 $(\mathrm{g}) /$ 体重 $(\mathrm{g})] \times 100$

肾上腺指数 $(\%)=[$ 肾上腺湿重 $(\mathrm{g}) /$ 体重 $(\mathrm{g})] \times 100$

脏器指数抑制率 $(\%)=[$ (给药组脏器指数 - 模型组脏器指 数) $/$ 模型组脏器指数 $] \times 100$

\section{Statistical analysis}

计量数据采用多因素方差分析结合Dunnett's多重比较法进行 统计分析。

\section{Results}

\section{Effect of Lanshi tonic on general physical signs in mice with kidney-yang deficiency}

正常组小鼠毛发润泽、活动自如; 连续注射氢化可的松琥 珀酸钠 7 天, 停止 3 天后, 模型组小鼠出现精神倦急、活动减 少、反应迟钝、皮感温度低、喜扎堆、蜷曲拱背、体毛干枯 稀疏无光泽, 爪甲与耳朵颜色变淡、饮食饮水减少等表现。 各组小鼠造模前体质量均一, 造模后模型组体重较正常组显著 下降 $(p<0.01)$ 。澜仕口服液各剂量组小鼠毛色和光泽度、迟 钝蜷缩表现有所改善, 各剂量组小鼠体质量均较模型组增加

Table 1. Effect of Lanshi tonic on body mass in mice with kidney-yang deficiency

\begin{tabular}{lcc}
\hline Group & Before model establishment (g) & After model establishment (g) \\
Normal group & $18.83 \pm 1.65^{1)}$ & $26.60 \pm 2.82$ \\
Model group & $18.92 \pm 1.88$ & $21.84 \pm 3.58^{\# \#}$ \\
High dose group & $18.95 \pm 1.92$ & $24.75 \pm 2.12^{* *}$ \\
Middle dose group & $19.25 \pm 1.61$ & $23.54 \pm 1.94^{*}$ \\
Low dose group & $19.04 \pm 1.84$ & $23.89 \pm 1.73^{*}$ \\
\hline
\end{tabular}

${ }^{1)}$ Mean \pm standard deviaiton; Compared with normal group, ${ }^{\# \#} p<0.01$; Compared with model group, ${ }^{*} p<0.05,{ }^{* *} p<0.01$. 
$(p<0.05, p<0.01)$ 。见Table 1 。

\section{Effect of Lanshi tonic on organ index in mice with kidney-yang deficiency}

连续注射氢化可的松琥珀酸钠 7 天, 停止 3 天后, 模型组 小鼠脾指数、胸腺指数、肾上腺指数均较正常组显著降低 $（ p<0.01 ）$; 灌胃给予澜仕口服液后, 各剂量组脾指数均较模 型组显著升高（ $p<0.05 ， p<0.01 ）$ ，其中澜仕口服液中剂量组脾 指数抑制率最高，达到 $25.99 \%$; 澜仕口服液高剂量组和中剂量 组胸腺指数及肾上腺指数均较模型组显著升高（ $p<0.05 ）$ ，其 中高剂量组胸腺指数抑制率和肾上腺指数抑制率最高, 分别达 到 $34.53 \%$ 、16.15\%。见Table 2 。

\section{Effect of Lanshi tonic on adrenal cortex function in mice with} kidney-yang deficiency

连续注射氢化可的松琥珀酸钠 7 天，停止 3 天后，模型组小 鼠血清cortisone、ACTH和尿17-0HCS含量均较正常组显著降低 $(p<0.05, p<0.01)$ 。澜仕口服液各剂量组血清Cortisone均较 模型组升高（ $p<0.05, p<0.01 ）$; 澜仕口服液高剂量组和中剂量 组血清ACTH均较模型组升高（ $p<0.05 ）$; 澜仕口服液各剂量组 尿17-0HCS含量较模型组均无显著变化 $(p>0.05)$ 。见Table 3 。

\section{Effect of Lanshi tonic on cyclic nucleotide level in mice with} kidney-yang deficiency

连续注射氢化可的松琥珀酸钠 7 天, 停止 3 天后, 与正常组 小鼠比较, 模型组小鼠血清cAMP含量降低, cGMP升高, cAMP/ cGMP比值降低（ $p<0.01 ）$ 。灌胃给予澜仕口服液后, 各剂量组 小鼠血清cAMP含量均升高, cGMP含量均降低, cAMP/cGMP比值 均升高 $(p<0.05, p<0.01)$ 。见Table 4 。

\section{Discussion}

肾阳虚者, 其温煦、生殖、气化功能下降, 以腰膝酸痛, 虚 寒及生殖能力下降为辨证要点 (Qin et al., 2017) 。临床患 者多见腰背酸痛、腰膝酸软、畏寒肢冷、嗜睡、耳鸣、耳狵、 面色萎黄、发脱枯悴、性机能异常等(Zhang et al., 1989)。 氢化可的松可升高血中糖皮质激素水平，抑制促肾上腺皮质激 素释放，而使类固醇激素分泌减少，出现一系列 “耗竭” 现象 (Qin et al., 2017; Dai et al., 2017), 特别是突然停用, 肾上腺轴的抑制状态立即暴露, 出现一系列阳虚表现 (Du et al. ，2010)。氢化可的松作为外源性糖皮质激素造成的肾阳虚 小鼠模型, 可导致神经-内分泌-免疫系统的功能变化, 从而引

Table 2. Effect of Lanshi tonic on organ index in mice with kidney-yang deficiency

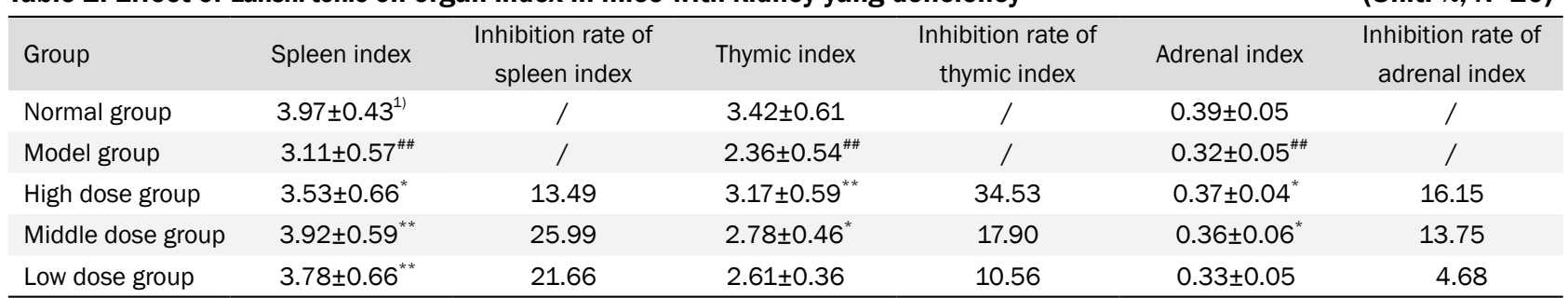

${ }^{1)}$ Mean \pm standard deviaiton; Compared with normal group, ${ }^{\# \#} p<0.01$; Compared with model group, ${ }^{*} p<0.05,{ }^{* *} p<0.01$.

Table 3. Effect of Lanshi tonic on serum cortisone, ACTH and 17-0HCS level in mice with kidney-yang deficiency

$(\mathbf{N}=\mathbf{2 0})$

\begin{tabular}{|c|c|c|c|}
\hline Group & Cortisone (ng/mL) & ACTH (pg/mL) & $17-\mathrm{OHCS}(\mathrm{nmol} / \mathrm{mL})$ \\
\hline Normal group & $13.05 \pm 4.75^{1)}$ & $1114.02 \pm 357.49$ & $75.72 \pm 9.66$ \\
\hline Model group & $8.84 \pm 0.99^{\# \#}$ & $915.96 \pm 142.04^{\#}$ & $60.64 \pm 9.08^{\# \#}$ \\
\hline Middle dose group & $10.34 \pm 1.44^{* *}$ & $1021.16 \pm 167.71^{*}$ & $64.58 \pm 9.06$ \\
\hline Low dose group & $9.84 \pm 1.72^{*}$ & $922.39 \pm 93.37$ & $64.90 \pm 7.81$ \\
\hline
\end{tabular}

${ }^{1)}$ Mean \pm standard deviaiton; Compared with normal group, ${ }^{\#} p<0.05,{ }^{\# \#} p<0.01$; Compared with model group, ${ }^{*} p<0.05,{ }^{*} p<0.01$.

Table 4. Effect of Lanshi tonic on cAMP and cGMP level and the ratio of cAMP/cGMP in mice with kidney-yang deficiency (N=20)

\begin{tabular}{|c|c|c|c|}
\hline Group & cAMP (nmol/L) & cGMP (nmol/L) & cAMP/cGMP \\
\hline Normal group & $141.29 \pm 15.83^{1)}$ & $139.88 \pm 22.50$ & $1.03 \pm 0.15$ \\
\hline Model group & $125.70 \pm 14.49^{\# \#}$ & $238.85 \pm 54.86^{\# \#}$ & $0.54 \pm 0.08^{\# \#}$ \\
\hline High dose group & $148.05 \pm 33.15^{*}$ & $137.56 \pm 34.46^{* *}$ & $1.13 \pm 0.35^{* *}$ \\
\hline Middle dose group & $146.03 \pm 24.60^{* *}$ & $151.37 \pm 33.09^{* *}$ & $1.00 \pm 0.21^{* *}$ \\
\hline Low dose group & $137.52 \pm 18.53^{*}$ & $166.96 \pm 20.23^{* *}$ & $0.83 \pm 0.09^{* *}$ \\
\hline
\end{tabular}

${ }^{1)}$ Mean \pm standard deviaiton; Compared with normal group, ${ }^{\#} p<0.05,{ }^{\# \#} p<0.01$; Compared with model group, ${ }^{*} p<0.05,{ }^{* *} p<0.01$. 
起内分泌器官肾上腺和免疫器官萎缩等 (Xu et al., 2015)。 环核苷酸是调节细胞功能活动的重要物质, 中医的阴阳学说 与环核苷酸理论有相似之处, 阳虚时主要是CGMP 含量上升, cAMP/cGMP 比值降低 (Chen, 1993)。有研究以大鼠cAMP 含量 下降, cGMP 含量显著升高及cAMP/cGMP比值显著降低作为肾阳 虚模型造模成功的评判标准 (Gou et al., 2009; Ren et al., 2010; Zhang et al., 2015)。

本实验研究显示, 肾阳虚模型小鼠出现一系列的 “耗竭”, 即体重下降、精神倦急、活动减少、反应迟钝、皮感温度低、 喜扎堆、蜷曲拱背、体毛干枯稀疏无光泽, 爪甲与耳朵颜色 变淡、饮食饮水减少等; 小鼠免疫器官脾、胸腺和内分泌器 官肾上腺均发生萎缩; 连续注射氢化可的松, 突然停用后, 肾上腺皮质功能受到抑制, 分泌激素减少, 小鼠血清ACTH、 Corti sone和尿17-0HCS含量均降低; 小鼠血清CAMP含量降低, cGMP升高, cAMP/cGMP比值降低, 符合肾阳虚动物模型鉴定标 准。本实验所用受试物澜仕口服液以黄芪和杜仲为君药, 可益 气补虚、补肝温肾; 以生地黄和巴戟天为臣药, 主入肾经, 生 髓填精; 以枸杞子和黄精为佐药, 平补肝肾精气、平补脾肾不 足; 全方配伍能温补肾阳、强筋健骨。灌胃给予肾阳虚模型 小鼠后, 可增加模型小鼠体重, 改善模型小鼠毛色、光泽度和 迟钝蜷缩等表现; 可升高肾阳虚模型小鼠脾指数、胸腺指数和 肾上腺指数; 可升高血清ACTH和cortisone含量; 可增加血清 cAMP含量, 降低cGMP含量, 升高cAMP/cGMP比值。

\section{Conclusion}

澜仕口服液在一定程度上具有改善肾阳虚小鼠虚损耗竭、抑 制免疫器官和内分泌器官萎缩的作用, 其作用机制可能通过改 善肾阳虚模型小鼠肾上腺皮质分泌功能、调整环核苷酸水平失 衡、恢复机体代谢平衡, 从而发挥对肾阳虚状态的调节作用。

\section{Author's contribution}

SL, YJS and HM designed the experiment and YJS, WYZ and JL operated the animal experiment. SL and SYM analyzed the data and YJS and HM wrote the article.

\section{Author details}

Shuo Liu (Engineer), Research and Development of Healthy Food, Infinitus (China) Co., Ltd., Hongtai Wisdom Valley, 19th of Si Cheng Rd, Tian He District, Guangzhou 510663, China; Si-yao Ma(Engineer), Research and Development of Healthy Food, Infinitus (China) Co., Ltd., Hongtai Wisdom Valley, 19th of Si Cheng Rd, Tian He
District, Guangzhou 510663, China; Wen-yi Zhu (Graduate student), Cosmetics Science and Technology, Beijing Technology and Business University, 11 Fucheng Road, Haidian District, Beijing 10048, China; Jia Liang (Graduate student), Traditional Chinese Medicine, Graduate School of Chinese Academy of Traditional Chinese Medicine, 16 Dongzhimen neinan street, Dongcheng District, Beijing 100700, China; Yu-jing Shi (Associate Research Fellow), Institute of Chinese Materia Medica, China Academy of Chinese Medical Sciences, Yinghua street, Chaoyang District, Beijing 110105, China; Hong Meng (Professor), Cosmetics Science and Technology, Beijing Technology and Business University, 11 Fucheng Road, Haidian District, Beijing 10048, China.

\section{References}

Chen XY. Practical animal model of TCM syndrome. Beijing, Peking Union Medical, pp108, 1993.

Dai B, Zhang JN, Yang ML, Li YX, Xiao ZZ, Yang L, Wei Q. Establishment of a mouse model of kidney deficiency induced by oral administration of hydrocortisone and evaluation of related factors. Acta Laboratorium Animalis Scientia Sinica, 25: 70-73, 2017.

Du J, Li N, Wang HM. Methods for preparing kidney deficient models and related evaluating factors. Journal of Clinical Rehabilitative Tissue Engineering Research, 14: 9433-9436, 2010.

Gou XJ, Han BX, Wang CT, Mu DH, Lu X, Zhang YY. Investigation of model-shaping method for deficiency syndrome of kidney-yang. Jilin Journal of Traditional Chinese Medicine, 29: 814-815, 2009.

Jia KJ, Ai X, Jia TZ, Ju CQ. Comparasion of antis egg-case before and after preparing in reproducing kidney-yang deficiency model rats. Journal of Chinese Medicinal Materials, 39: 1516-1520, 2016.

Lin ZJ, Zhang B, Liu XQ, Zhu WJ. Research on animal model evaluation with traditional Chinese medicine principles. China Journal of Traditional Chinese Medicine and Pharmacy, 28: 2217-2221, 2013.

Qin WY, Chen H, Zhu JH, Qi Y, Zhao JM. Comparison of the different model establishment methods of kidney yang deficiency in mice caused by hydro-cortisone. Laboratory 
Animal Science, 34: 11-14, 2017.

Ren YS, Zhao YL, Wang JB, Zhang P, Zhao HP, Zhang XR, Zhou CP, Xiao XH. Investigation on cold/heat characters of kidney yin/yang deficiency models by zoogenic thermal activity. Chinese Journal of Experimental Traditional Medical Formulae, 16: 94-101, 2010.

Shen ZY. Study on the location of kidney-yang deficiency syndrome. Chinese Journal of Integrated Traditional and Western Medicine, 17: 50-52, 1997.

Xu HF, Yang S, Li SS, Li TT, XU XF, Li XR. The effect on immunological function of rat modle with kidney-yang deficiency syndrome of ethanol extracts of Cuscuta chinensis Lam. Journal of Chinese Medicinal Materials, 38: 2163-2165, 2015.
Zhang D, Li Z, Zhu QJ, Li Z, Tao HH. Establishment and evaluation in mice model with kidney-yang deficiency syndrome caused by overfating and sexual overstrain. Laboratory Animal Science, 25: 9-11, 2008.

Zhang QY, Wu ZM, Feng YS, Shi JD. The relationship between the determination of sex hormone and syndrome differentiation in patients with chronic renal failure. Journal of Traditional Chinese Medicine, 287: 30-31, 1989.

Zhang ZY, Chen BJ, Zhang YJ, Wang WT, Wang YR, Su YX. Establishment and stability observation of rat model of kidney-yang deficiency and kidney-yin deficiency. Fujian Journal of Traditional Chinese Medicine, 46: 51-54, 2015. 


\section{中文摘要}

\section{澜仕口服液对肾阳虚小鼠模型的调节作用研究}

刘硕 ${ }^{1}$, 马思遥 ${ }^{1}$, 朱文驿 ${ }^{2}$, 梁佳 $^{3}$, 时宇静 ${ }^{4}$, 孟宏 $^{*}$

${ }^{1}$ 无限极（中国）有限公司健康食品研发, 广州, 中国

${ }^{2}$ 北京工商大学化妆品科学与技术, 北京, 中国

${ }^{3}$ 中国中医科学院研究生院中医学, 北京, 中国

${ }^{4}$ 中国中医科学院中药研究所, 北京, 中国

目的: 研究澜仕口服液对肾阳虚状态的调节作用及相关机制。方法: 将ICR小鼠按体质量随机分为 5 组: 正常组、 模型组、澜仕口服液高、中、低剂量组。小鼠腹腔注射氢化可的松琥珀酸钠, $50 \mathrm{mg} / \mathrm{kg}$, 连续 7 天, 停止注射 3 天造成肾阳虚证候小鼠模型。造模同时给予各剂量澜仕口服液，正常组和模型组在同等条件下给予蒸馏水。观 察并记录小鼠体质量、毛色、体态等一般体征状态, 解剖、称量并计算脾指数、胸腺指数和肾上腺指数, ELISA 法检测尿液17-OHCS含量, ELISA法检测血清Cortisol、ACTH、CAMP、cGMP含量、计算CAMP/cGMP比值。结 果: 与正常组比较, 模型组小鼠除血清CGMP显著升高外, 上述其他指标均显著降低 $(p<0.05, p<0.01)$ 。与模 型组比较, 澜仕口服液高剂量和中剂量除对尿液 $17-\mathrm{OHCS}$ 含量无显著改变（ $p>0.05 ）$ 外, 对上述其他指标均有 不同程度的改善作用 $(p<0.05, p<0.01)$ 。结论: 澜仕口服液对肾阳虚状态具有调节作用, 可能与调节肾上腺皮 质分泌功能和调整环核苷酸水平失衡相关。

关键词: 澜仕口服液, 氢化可的松琥珀酸钠, 肾阳虚, 肾上腺皮质, 环核苷酸 


\section{국문초록}

\section{신장 양 결핍 마우스 모델에 대한 Lanshi 토닉의 조절 효능}

류석 ${ }^{1}$, 마사요 ${ }^{1}$, 주문이 ${ }^{2}$, 양가 $^{3}$, 시우정 ${ }^{4}$ 맹홍 ${ }^{2 *}$

${ }^{1}$ 무한급(중국)유한회사 건강식품개발, 광주, 중국

${ }^{2}$ 북경공상대학 화장품과학과 기술, 북경, 중국

3중국중의과학원연구생원 중의학, 북경, 중국

${ }^{4}$ 중국중의과학원중약연구소, 북경, 중국

목적: 신장 양 결핍 상태에 대한 란시(Lanshi)토닉의 조절 효과 및 관련 메커니즘 연구하고자 한다. 방법: ICR 마우스는 체중에 따 라 무작위로 정상 군, 모델 군, 란시 토닉 고용량, 중용량 및 저용량 그룹 등 5 개 그룹으로 분류되었다. 마우스모델에게 연속적으로 7 일 동안 $50 \mathrm{mg} / \mathrm{kg}$ hydrocortisone sodium succinate를 복강 내 주사하고, 신장-양 결핍 증후군의 마우스 모델을 유발시키기 위 해 주사를 3 일 동안 중단시켰다. 동시에, 모든 용량의 란시 토닉을 각 신장-양 결핍 증후군의 마우스 모델에 각각의 란시 토닉을 투여하고 정상 그룹 및 모델 그룹에 동일한 조건 하에서 증류수를 제공 하였다. 마우스의 체질량, 모발 색 및 체형을 관찰하고 기록 하였고, 비장 지수, 흥선 지수 및 부신 지수를 계산 하였다. ELISA로 소변 내17-OHCS 함량 측정 및 혈청 코티솔, ACTH, cAMP, $\mathrm{cGMP}$ 의 함량을 측정하고 $\mathrm{cAMP} / \mathrm{cGMP}$ 의 비율을 계산하였다. 결과: 정상 그룹과 비교하여, 혈청 $\mathrm{cGMP}$ 가 유의하게 더 높은 경우 를 제외하고 $(p<0.01)$, 모델 그룹 마우스의 상기 지수는 유의하게 더 낮았다 $(p<0.05, p\langle 0.01)$. 모델 그룹과 비교하여, 고용량 및 중용 량의 란시 경구용액은 소변 17-OHCS 함량에서 유의 한 변화가 없었지만( $p>0.05)$, 상기 언급 된 다른 지표에 대해 상이한 개선 효 과를 가졌다 $(p<0.05, p<0.01)$. 결론: Lanshi 토닉은 신장 양 결핍 상태에 대한 조절 효과를 가지며, 이는 부신 피질의 분비 기능 조 절 및 사이 클릭 뉴클레오티드 수준의 불균형 조정과 관련 될 수 있다고 사료된다.

핵심어: 란시토닉, 박산히드로코르티손나트륨, 신장 양 결핍, 부신피질, 고리형 뉴클레오타이드 
